\title{
Relación costo-efectividad de la vacuna contra Haemophilus influenzae tipo b en niños menores de dos años de edad en Colombia
}

\author{
Nelson Alvis Guzmán, ${ }^{1}$ Fernando De La Hoz Restrepo ${ }^{2}$ \\ y David Vivas Consuelo 3
}

Forma de citar

Alvis Guzmán N, De La Hoz Restrepo F, Vivas Consuelo D. Relación costo-efectividad de la vacuna contra Haemophilus influenzae tipo b en niños menores de dos años de edad en Colombia. Rev Panam Salud Publica. 2006;20(4):248-55.

RESUMEN Objetivos. Las vacunas conjugadas contra Haemophilus influenzae tipo b (Hib) son la herramienta más importante para prevenir la mayoría de las enfermedades invasoras producidas por dicho patógeno, pero debido a su costo, aún no se han introducido mundialmente de manera masiva. En el presente estudio se determinó la relación costo-efectividad de una vacuna contra Hib para prevenir la neumonía y la meningitis bacterianas en niños menores de 2 años en Colombia.

Métodos. Se estimaron los costos directos e indirectos de la neumonía y la meningitis hospitalaria y siguiendo las recomendaciones de la Organización Mundial de la Salud (OMS), la relación costo-efectividad de los programas de vacunación contra Hib. Se estimaron también las razones de costos por caso evitado de enfermedad invasora por Hib y el costo por año de vida salvado en dos situaciones hipotéticas: con vacunación contra Hib (cobertura vacunal: 90\%) y sin vacunación.

Resultados. El costo medio del tratamiento hospitalario de un caso de neumonía fue de 611,5 dólares estadounidenses (US\$) (intervalo de confianza del 95\% [IC95\%]: 532,2 $690,8)$, el costo medio del tratamiento hospitalario de un caso de meningitis fue de US\$ 848,9 (IC95\%: 716,8 - 981,0) y el costo por caso evitado de enfermedad invasora por Hib, de US\$ 316,7 (IC95\%: 294,2 - 339,2). La relación costo-efectividad en la hipótesis con vacunación fue de 2,38, frente a 3,81 en la hipótesis sin vacunación.

Conclusión. La aplicación de un programa adecuado de vacunación contra Hib en Colombia puede prevenir cerca de 25000 casos de enfermedad invasora por año, lo que representa un ahorro de por lo menos US\$ 15 millones anuales. Además, puede evitar cerca de 700 defunciones y salvar anualmente 44054 años de vida.

Palabras clave Análisis económico, costo efectividad, Haemophilus influenzae tipo b, vacunas contra Haemophilus, Colombia.

Departamento de Investigaciones Económicas y Sociales (DIES), Facultad de Ciencias Económicas, Universidad de Cartagena. Toda correspondencia deberá dirigirse a: Nelson Alvis Guzmán, Crespo Calle 70 N. ${ }^{\circ} 7-33$, Cartagena, Colombia. Teléfono: (57 5) 6568031; correo electrónico: nalvis@yahoo. com

2 Universidad Nacional de Colombia, Instituto Nacional de Salud, Bogotá D.C., Colombia.
Las infecciones respiratorias agudas son uno de los problemas de salud pública más importantes en los países en

\footnotetext{
3 Centro de Investigación en Economía y Gestión de las Salud. Universidad Politécnica de Valencia, Valencia, España.
}

desarrollo, donde constituyen una de las primeras cinco causas de mortalidad en niños menores de 5 años y la primera causa de consulta y hospitalización en los servicios de pediatría (1). Se estima que en 2002 , se produjeron 
10,2 millones de muertes en menores de 5 años en el mundo, de las cuales, 1856000 se atribuyeron a infecciones respiratorias agudas (neumonía en la inmensa mayoría de los casos) (2). En países en desarrollo, diversos estudios han demostrado que más de la mitad de los casos de neumonía están producidos por bacterias, entre las que predominan Streptococcus pneumoniae (30\%) y Haemophilus influenzae tipo b (Hib) (27\%). Esto implica que la mayoría de las defunciones por neumonía en niños pequeños se deben a infecciones por estos patógenos (3). Hib es el principal agente causal de la meningitis bacteriana endémica en la población infantil de los países de América, incluida Colombia. Se estima que en los países en desarrollo, Hib causa hasta $30 \%$ de los casos de neumonía con cultivo positivo y entre $20 \%$ y $60 \%$ de los casos de meningitis bacteriana, con una letalidad cercana al $40 \%$. Hib ocasiona además unos 50000 casos anuales de meningitis, de los cuales, $90 \%$ ocurre en niños menores de 5 años, con un máximo entre los 6 y los 11 meses de edad (4-6).

La neumonía bacteriana por Hib es muy frecuente en niños menores de 2 años. Se estima que este patógeno causa la mitad de los casos de neumonía que motivan ingresos en centros de tercer nivel de atención $(7,8)$. Según las estadísticas del Ministerio de la Protección Social de Colombia, en 2001, en este país, la neumonía causó la muerte de alrededor de 5000 niños menores de 5 años y el ingreso hospitalario de aproximadamente 80000 niños, mientras que la meningitis ocasionó la muerte de alrededor de 600 niños menores de 5 años y el ingreso de aproximadamente 3000 niños (9).

Desde hace unos años está disponible en el mercado una vacuna contra el Hib de eficacia demostrada en la prevención de las enfermedades causadas por este microorganismo. Estos avances deben cambiar radicalmente el enfoque de los programas de prevención de las infecciones respiratorias (10). La introducción de esta vacuna conjugada ha reducido la incidencia de la enfermedad invasora por Hib en $95 \%$ o más en los países industrializados $(11,12)$.
La demostración de la eficacia y la inocuidad de esta vacuna (13-16) ha motivado su inclusión en los programas nacionales de inmunización infantil de la mayoría de los países desarrollados. No ha ocurrido lo mismo en los países en desarrollo, donde el alto costo relativo de esta vacuna ha sido el principal obstáculo para su administración regular en el marco de programas de inmunización. En efecto, dicho costo supera con creces el de las vacunas básicas del Programa Ampliado de Inmunización (PAI), es decir, las vacunas contra la difteria, la tos ferina y el tétano (vacuna combinada DPT), la poliomielitis, el sarampión y la hepatitis B.

Para revertir esta situación, la Organización Mundial de la Salud (OMS) resalta la necesidad de alcanzar compromisos políticos que procuren la financiación suficiente para garantizar la continuidad de los programas de inmunización una vez iniciados.

Para demostrar la necesidad de incluir esta vacuna en los programas públicos de inmunización de los países en desarrollo, hay que evidenciar que los beneficios de la vacunación superan a los costos. Este es el enfoque de la evaluación económica de los programas de inmunización. Varios estudios han analizado el impacto de la vacuna contra Hib en la frecuencia de neumonía y meningitis bacterianas (17-20). Tomando como referencia estas investigaciones, se presentan en este artículo los resultados de un estudio de evaluación económica realizado en Colombia. El enfoque que hemos utilizado para esta evaluación es el análisis de costo-efectividad (21).

\section{MATERIALES Y MÉTODOS}

En el diseño del estudio se tuvieron en cuenta las recomendaciones de la OMS de 2001 respecto de la estimación de la relación costo-efectividad de la vacunación contra Hib a partir de la confrontación de la carga estimada de enfermedad con los costos. El análisis se realizó desde dos perspectivas: el sector sanitario y la sociedad. Para ello, se determinaron dos tipos de costos: a) los costos de la vacuna y de su administración, y b) los costos de los casos de enfermedad evitados como consecuencia de la vacunación. En el análisis desde la perspectiva de la sociedad, se estimaron asimismo los gastos y los costos derivados del tiempo dedicado a los niños por familiares o cuidadores, durante la enfermedad o la vacunación. Sin embargo, dadas las dificultades para obtener estos datos, la OMS recomienda limitar el análisis a la perspectiva del sector sanitario (22-25).

La investigación se planteó en dos fases. En la primera, iniciada en 2001, se fortalecieron los procedimientos de vigilancia epidemiológica de la neumonía y la meningitis bacterianas en niños menores de 2 años ingresados en centros hospitalarios pediátricos de tres de las cinco principales ciudades colombianas (Hospital Infantil Napoleón Franco Pareja de Cartagena; Clínica del Niño y Departamento de Pediatría del Hospital Simón Bolívar, de Bogotá; y Hospital San Vicente de Paúl de Medellín). Esto permitió la captura de la información sobre costos de atención y la valoración de los resultados. De igual modo se pudieron establecer los costos asociados a la vacunación contra Hib. Se diseñó y estandarizó una ficha de vigilancia epidemiológica de la neumonía y la meningitis (VENM), que se adoptó como instrumento regular de captura de datos en los hospitales seleccionados para el estudio. Los criterios de selección de los hospitales fueron: a) ser un hospital pediátrico de remisión de casos (tercer nivel) de neumonía y meningitis para su área de influencia (Bogotá, Medellín o Cartagena, ciudades que agrupan un poco más de $25 \%$ de la población colombiana y se hallan en regiones geográficas disímiles), b) ser un centro de formación de recursos humanos (médicos especialistas), lo cual contribuye a un mejor seguimiento de los casos, y c) contar con la infraestructura tecnológica adecuada para el diagnóstico y el tratamiento de la neumonía y la meningitis.

Los casos de neumonía y meningitis se seleccionaron de manera secuencial entre los niños atendidos en dichos hospitales entre julio de 2001 y diciem- 
bre de 2002 que cumplían los criterios de inclusión: a) se incluyó entre los casos de neumonía bacteriana a todo niño menor de 2 años hospitalizado en alguno de los centros del estudio con diagnostico clínico de neumonía y radiografía de tórax con patrón sugestivo de neumonía bacteriana, refrendado por un radiólogo experto; b) se incluyó entre los casos de meningitis bacteriana a todo niño menor de 2 años que consultó por fiebre alta acompañada de al menos uno de los siguientes signos: convulsiones, rigidez de nuca y coma, además de alteraciones en la muestra de líquido cefalorraquídeo sugestivas de meningitis bacteriana (disminución de la concentración de glucosa, aumento del número de leucocitos polimorfonucleares o detección de bacterias grampositivas) o cultivo de Hib positivo.

Para el análisis de los costos generados por la enfermedad, se determinaron los costos directos e indirectos asociados con los distintos casos. La estimación de los costos directos abarcó el tratamiento hospitalario, para lo cual se identificaron los procesos de atención en lo relativo a la estancia, el tratamiento farmacológico y el diagnóstico, y se determinó la variabilidad de los costos por caso. La información sobre los costos directos fue suministrada y validada por los sistemas de costos de los hospitales respectivos.

Para calcular los costos indirectos se tuvo en cuenta, en cada caso, la pérdida de productividad del familiar encargado de acompañar al niño durante la hospitalización o las visitas para recibir atención sanitaria. Esta pérdida se estimó según la actividad del acompañante, el ingreso mensual del hogar, los costos de transporte y el tiempo de espera para recibir la atención.

La información sobre los costos se integró y procesó con el programa EPI INFO 2002 y en una hoja de cálculo de MS Excel 2000. El análisis de esta información permitió establecer las descripciones estadísticas y estimar los costos medios por caso de neumonía y por caso de meningitis, con sus respectivos intervalos de confianza de $95 \%$. De igual modo se tabularon y se procesaron los datos sobre los costos directos e indirectos de la vacunación. A partir del costo medio de atención por caso de neumonía y por caso de meningitis, y del costo de administrar la vacuna, se calcularon los costos totales, tanto en una situación sin vacunación como en una situación con una cobertura vacunal de 90\%. La información sobre la efectividad de la vacuna respecto a la neumonía y a la meningitis se obtuvo de estudios anteriores realizados en Colombia (19).

El costo medio de la administración de la vacuna se determinó valorando el proceso de vacunación de los niños en cada ciudad del estudio. Los costos directos están relacionados con la adquisición, la importación, la gestión y el transporte de la vacuna a la ciudad respectiva. Además se estimó el costo de aplicación teniendo en cuenta principalmente los costos de personal, representados por los salarios. A partir de los datos obtenidos, se elaboraron cuadros donde se especifican tanto las medidas de efectividad como los costos de las distintas intervenciones. El análisis del costo medio de la atención de un caso de neumonía y de un caso meningitis, y de la aplicación de la vacuna, conocidas las estimaciones de su eficacia, se realizó comparando los costos totales de las acciones alternativas, expresados en términos de costo medio por caso.

En la segunda fase del estudio, se plantearon dos situaciones hipotéticas: en una de ellas, se administra la vacuna contra Hib a la población de niños menores de 2 años, y en la otra, no. Para ambas cohortes se estimaron las incidencias de la neumonía y la meningitis bacterianas $(19,20)$ y se valoraron los respectivos costos del tratamiento hospitalario. Luego se determinaron los casos evitados y los costos respectivos, lo cual permitió establecer la relación costo-efectividad de la vacunación contra Hib frente a la alternativa sin vacunación. Las estimaciones de las incidencias de ambas enfermedades se basaron en los resultados de los estudios de carga de la enfermedad que el Ministerio de Salud de Colombia llevó a cabo en 1990 y 1995 (26).

A partir de los números de casos previstos de neumonía y de meningitis por
Hib, basados en las tasas de incidencia respectivas, y de los costos medios, se calcularon las razones medias de costos por caso tratado de neumonía y de meningitis, y las razones medias de costos por caso evitado de enfermedad invasora por Hib. Además, extrapolando las proporciones de mortalidad obtenidas en el estudio al número de casos previstos, se estimó el número de años de vida salvados (AVS) con el tratamiento hospitalario de las dos enfermedades y con la aplicación del programa de vacunación. Se utilizó el número de años de vida salvados como medida de efectividad debido a su sensibilidad para valorar el impacto de las intervenciones en la mortalidad.

El análisis se realizó según las siguientes características:

- Se consideraron dos situaciones hipotéticas: población vacunada y no vacunada.

- El horizonte temporal considerado fue el año 2002.

- La situación epidemiológica considerada fue la actual, reflejada en el riesgo anual derivado de los estudios realizados por el Instituto Nacional de Salud de Colombia.

- En la hipótesis con vacunación, los costos resultaron de sumar los costos de atención relativos a los casos no evitados y los costos del programa de vacunación que garantiza una cobertura del $90 \%$ y una efectividad del $90 \%$ respecto del la meningitis y del 53\% respecto de la neumonía.

- Las proporciones de mortalidad de la meningitis y la neumonía bacterianas consideradas en este estudio fueron las encontradas en los hospitales participantes.

- Los números de años de vida salvados se calcularon basándose en una esperanza de vida de 72,2 años, según las estimaciones para Colombia en el año 2002.

- La tasa representativa del mercado (TRM) para el cambio del dólar estadounidense se fijó en 2727,5 pesos por dólar.

- Por último, se realizó un análisis de sensibilidad en el que se tuvo en cuenta la variabilidad de la incidencia de la infección por Hib, la cual 
depende de la efectividad de la vacuna. De esta manera, se generó un intervalo de efectividad de la vacuna comprendido entre $50 \%$ y $99 \%$, lo que supone probabilidades decrecientes de infección por Hib.

\section{RESULTADOS}

\section{Costos directos e indirectos}

Para analizar los costos se estudiaron 64 casos de neumonía y 25 casos de meningitis que cumplieron con los criterios de inclusión señalados en el protocolo. El resultado de los análisis de los costos medios de la neumonía y la meningitis bacterianas se resume en el cuadro 1, donde se evidencia el predominio de los costos directos, con estructuras de composición similares para las dos enfermedades. Los costos indirectos representaron $11 \%$ del total en el caso de la neumonía y $14 \%$ en el de la meningitis, sin que se hayan observado diferencias significativas, probablemente por las semejanzas en los ingresos que dejaron de recibir los familiares acompañantes.

El costo medio de la vacunación (adquisición y aplicación de la vacuna contra Hib) fue de US\$ 9,3 (IC95\%: $8,6-10,1)$ para las tres dosis. El costo de adquisición equivalió a nueve veces el costo de administración.

\section{Costo-efectividad}

La efectividad de tres dosis de la vacuna contra Hib se estimó en 53\% respecto de la neumonía bacteriana (21) y entre $90 \%$ y $99 \%$ respecto de la meningitis $(3,19)$. El número de casos previstos de neumonía por Hib correspondió a las estimaciones de De La Hoz et al (20) y el de meningitis, al número de casos registrados en el Sistema de Vigilancia Epidemiológica del Ministerio de Salud de Colombia (SIVIGILA) en 2001. Con estos supuestos, la vacunación con el esquema completo contra Hib representa un costo por caso evitado de enfermedad invasora de US\$ 316,7, mientras que el costo del tratamiento hospitalario por

CUADRO 1. Costos directos e indirectos de atención de la neumonía y la meningitis bacterianas por Haemophilus influenzae, ${ }^{a}$ Colombia, 2002

\begin{tabular}{|c|c|c|c|c|}
\hline Costos & $\begin{array}{c}\text { Neumonía } \\
\text { bacteriana } \\
(25 \text { casos) } \\
(\text { US } \$)^{b}\end{array}$ & $\begin{array}{l}\text { IC95\% }{ }^{\mathrm{C}} \\
\text { (US\$) }^{\mathrm{b}}\end{array}$ & $\begin{array}{c}\text { Meningitis } \\
\text { bacteriana } \\
(26 \text { casos) } \\
(\text { US } \$)^{b}\end{array}$ & $\begin{array}{l}\text { IC95\% }{ }^{\mathrm{C}} \\
\text { US\$) }^{\mathrm{b}}\end{array}$ \\
\hline Estancia hospitalaria & 329,1 & $284,2-374,0$ & 552,9 & $470,6-635,2$ \\
\hline Medicamentos & 58,4 & $42,4-74,5$ & 86,0 & $50,9-121,1$ \\
\hline Antibióticos & 45,3 & $30,1-60,5$ & 75,8 & $39,0-112,7$ \\
\hline Anticonvulsivos & - & - & 6,8 & $4,6-9,1$ \\
\hline Otros medicamentos & 14,1 & $10,3-17,9$ & 9,4 & $7,0-11,7$ \\
\hline Pruebas diagnósticas & 72,2 & $57,0-87,5$ & 130,8 & $113,6-148,1$ \\
\hline Exámenes básicos de laboratoriod & 49,9 & $38,7-61,0$ & 68,6 & $60,5-76,7$ \\
\hline Radiología & 18,9 & $14,5-23,3$ & 66,4 & $57,1-75,7$ \\
\hline Otros exámenes de laboratorio & 15,2 & $11,8-18,6$ & 17,9 & $13,8-22,1$ \\
\hline Servicios especiales & 128,5 & $102,9-154,0$ & 160,2 & $102,2-218,2$ \\
\hline Costos medios directos & 562,9 & $484,2-641,5$ & 826,3 & $701,0-951,7$ \\
\hline Costos medios indirectos & 67,6 & $59,2-76,1$ & 118,4 & $94,4-142,4$ \\
\hline Costo medio total & 611,5 & $532,2-690,8$ & 848,9 & $716,8-981,0$ \\
\hline
\end{tabular}

Fuentes: Refs. 26-28.

a En Hospital Infantil Napoleón Franco Pareja (Cartagena), Clínica del Niño y Departamento de Pediatría del Hospital Simón Bolívar (Bogotá) y Hospital San Vicente de Paúl (Medellín).

${ }^{b}$ Los valores se expresan en dólares estadounidenses [US\$] de 2002.

c IC95\%: intervalo de confianza del $95 \%$.

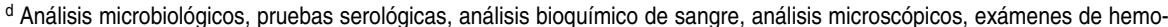
clasificación y pruebas previas a la transfusión de sangre y hemoderivados, análisis de biopsias y determinación de gases en sangre.

caso es de US\$611,5 para la neumonía bacteriana y de US\$ 848,9 para la meningitis bacteriana.

Como se indica en el cuadro 2, en la hipótesis en la cual se aplica el programa de vacunación se genera un ahorro (beneficio) de atención hospitalaria de casos de más de US $\$ 8,6$ millones. Aproximadamente $90 \%$ de este ahorro corresponde a costos directos y $10 \%$, a costos indirectos, lo cual implica un beneficio, desde la perspectiva social, de US\$ 0,86 millones.

La efectividad se evaluó mediante el número de años de vida salvados en las dos hipótesis — sin vacunación contra Hib y con vacunación-, tomando como año de referencia para los cálculos el año 2002. Se observó mayor efectividad de la hipótesis con vacunación contra Hib, en la cual se generaron 25194 años de vida salvados adicionales al evitarse 360 muertes por enfermedad invasora (cuadro 3). Esto es más notorio en el caso de la meningitis, dada su mayor gravedad.

La relación costo-efectividad en la situación con vacunación es de US\$ 2,38 por año de vida salvado, frente a US\$ 3,81 en la situación sin vacunación. La relación costoefectividad marginal evidencia una franca relación dominante de la estrategia de vacunación respecto a la situación sin vacunación (cuadro 4).

\section{Análisis de sensibilidad}

Las variaciones de las probabilidades de infección del orden del $40 \%$, resultantes de la variabilidad de la efectividad de la vacuna, no tienen efecto en la relación costo-efectividad. Aun con el mayor valor de probabilidad de infección dentro del intervalo considerado - que es cercano al de la probabilidad en la situación sin vacunaciónno se consigue equilibrar la relación costo-efectividad de la vacuna, es decir, el costo por año de vida salvado en la estrategia con vacunación sigue siendo inferior al de la situación sin vacunación (figura 1). Al disminuir la incidencia de la enfermedad, disminuye la diferencia entre las relaciones costo-efectividad en las dos hipótesis consideradas - con y sin vacunación-, lo cual sugiere que deberían producirse reducciones muy grandes de la incidencia para observar un punto de intercepción de las dos hipótesis (incidencia de la neumonía $<80$ por 100 000) (figura 2). Ahora 
CUADRO 2. Impacto de la vacunación en la reducción de los costos del tratamiento hospitalario de la neumonía y la meningitis bacterianas, Colombia, 2002 (estimación de las incidencias basadas en estudios de carga de la enfermedad realizados por el Ministerio de Salud de Colombia)

\begin{tabular}{|c|c|c|}
\hline & $\begin{array}{l}\text { Costo }^{\mathrm{a}} \\
\text { (US\$) }\end{array}$ & $\begin{array}{l}\text { IC95\% } \\
\text { (US\$) }\end{array}$ \\
\hline \multicolumn{3}{|l|}{ A. Hipótesis sin vacunación contra Hib ${ }^{\mathrm{b}}$ (casos previstos en 2002) } \\
\hline Costo total de la atención hospitalaria por enfermedad invasora por Hib & 31996554 & $29309923-36182194$ \\
\hline \multicolumn{3}{|l|}{ B. Hipótesis con vacunación contra Hib (casos evitados con $90 \%$ de cobertura) } \\
\hline Costo total evitado (ahorro) de atención hospitalaria por enfermedad invasora por Hib & 14512016 & $13331406-16397627$ \\
\hline Costo de los 26500 casos evitados de neumonía ${ }^{c}$ (US\$ 611,5/caso) & 16204750 & $14898300-18306200$ \\
\hline Costo de los 1507 casos evitados de meningitis ${ }^{d}$ (US $\$ 848,9 /$ caso) & 1279292 & $1080218-1478367$ \\
\hline Costo total evitado (ahorro) de atención por enfermedad invasora por Hib & 17484042 & 15978 518-19784567 \\
\hline Costo de la aplicación del esquema de vacunación contra Hib a 950000 niños (US\$9,4/esquema) & 8868810 & $8170000-9595000$ \\
\hline Ahorro neto con el esquema de vacunación contra Hib & 8615232 & $7808518-10189567$ \\
\hline
\end{tabular}

Fuentes: Refs. 19, 20, 26.

a Los valores se expresan en dólares estadounidenses (US\$) de 2002.

${ }^{\mathrm{b}}$ Hib: Haemophilus influenzae tipo b.

c Efectividad de la vacuna: $53 \%$.

d Efectividad de la vacuna: $90 \%$.

CUADRO 3. Número de años de vida salvados (AVS) en las hipótesis sin y con vacunación contra Haemophilus influenzae tipo b (Hib)

\begin{tabular}{|c|c|c|c|c|}
\hline & $N$ & $\begin{array}{c}\text { Proporción } \\
\text { de mortalidad }\end{array}$ & $\mathrm{N} .^{\circ}$ de muertes & $\mathrm{N} .{ }^{\circ}$ de AVS \\
\hline \multicolumn{5}{|l|}{ Hipótesis sin vacunación contra Hib } \\
\hline Casos previstos de neumonía & 50000 & 0,0054 & 273 & 3590890 \\
\hline Casos previstos de meningitis & 1674 & 0,1430 & 239 & 104123 \\
\hline Total & & & 512 & 3695013 \\
\hline \multicolumn{5}{|l|}{ Hipótesis con vacunación contra Hib } \\
\hline Casos previstos de neumonía & 23500 & 0,0054 & 128 & 3601018 \\
\hline Casos previstos de meningitis & 1674 & 0,1430 & 24 & 119189 \\
\hline Total & & & 152 & 3720207 \\
\hline Diferencia de AVS entre las hipótesis & & & 360 & 25194 \\
\hline
\end{tabular}

a Las proporciones de mortalidad de la meningitis y la neumonía bacterianas corresponden a las encontradas en los hospitales participantes en el estudio.

CUADRO 4. Resultados del análisis de costo-efectividad, hipótesis sin y con vacunación contra Haemophilus influenzae tipo b (Hib)

\begin{tabular}{lcccccc}
\hline Estrategia & $\begin{array}{c}\text { Costo } \\
\text { (US\$) }\end{array}$ & $\begin{array}{c}\text { Costo } \\
\text { marginal } \\
\text { (US\$) }\end{array}$ & $\begin{array}{c}\text { Efectividad } \\
\left.\text { (AVS }^{b}\right)\end{array}$ & $\begin{array}{c}\text { Efectividad } \\
\text { marginal } \\
\text { (AVS) }\end{array}$ & $\begin{array}{c}\text { Relación } \\
\text { costo- } \\
\text { efectividad } \\
\text { (US\$/AVS) }\end{array}$ & $\begin{array}{c}\text { Relación } \\
\text { costo-efectividad } \\
\text { marginal } \\
\text { (US\$/AVS) }\end{array}$ \\
\hline Con vacunación & 3,18 & 3,27 & $\begin{array}{c}1,693 \\
1,337\end{array}$ & 0,357 & 2,38 & 9,20 \\
Sin vacunación & 6,45 & & & 3,81 &
\end{tabular}

a Los valores se expresan en dólares estadounidenses (US\$) de 2002.

${ }^{b}$ AVS: años de vida salvados.

bien, las tasas de este tipo son inverosímiles en países como Colombia.

Cuando se realiza un análisis de sensibilidad de doble vía, es decir, considerando variaciones en la inci- dencia de la enfermedad y en la efectividad de la vacuna, se observa que la relación costo-efectividad en la hipótesis con vacunación es mucho mayor, con incidencias de más de 400 por
100000 y efectividad de la vacuna superior a $70 \%$.

\section{DISCUSIÓN}

El índice de costo-efectividad empleado en este estudio - costo por año de vida salvado- permite comparar los resultados obtenidos con los de otras actividades de prevención y evaluar otros programas de vacunación contra enfermedades con igual o mayor mortalidad. Los resultados se pueden presentar en términos de costo o de ahorro por año de vida salvado. Esto es importante, dado que el objetivo de un programa de vacunación sistemática para controlar la enfermedad invasora por Hib no solo es evitar el sufrimiento y prevenir las complicaciones graves de la enfermedad, sino también salvar vidas. Por ello, al utilizar el número de años de vida salvados como medida de efectividad en el caso de enfermedades inmunoprevenibles de baja letalidad, se puede subestimar la relación costoefectividad de dicho programa. La vacuna contra Hib ha sido evaluada económicamente utilizando esta medida de efectividad, en especial en países desarrollados (29-33).

Varias son las razones por las cuales pensamos que los resultados de nuestro estudio, pese a ser importantes, su- 
FIGURA 1. Análisis de sensibilidad: variación del costo por año de vida salvado (AVS) en función de la efectividad de la vacuna contra Haemophilus influenzae tipo b (Hib) (probabilidad de infección)

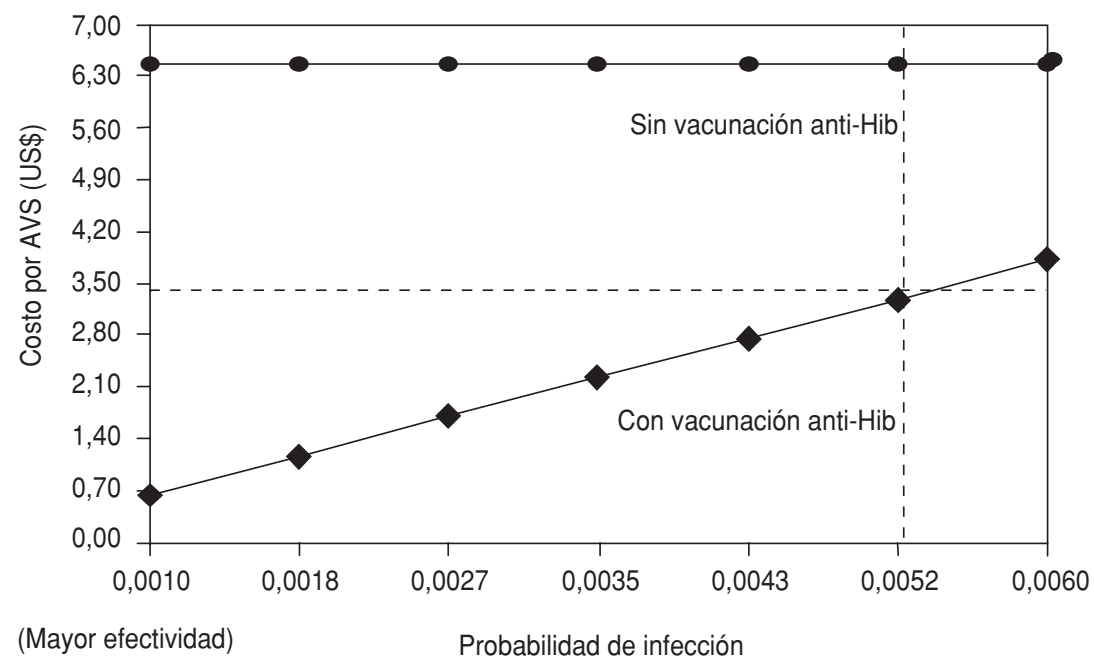

bestiman la relación costo-beneficio de la introducción de la vacuna contra Hib. Por un lado, debido a la falta de información, no se pudieron incluir los costos de la contención de epidemias, del tratamiento de las secuelas crónicas de la meningitis y de la atención de pacientes ambulatorios con neumonía, los cuales representan la mayoría de los casos de neumonía. Por otro, dado que los hospitales participantes en el estudio atienden a personas de niveles socioeconómicos bajos, los costos indirectos considerados corresponden a la pérdida de productividad de hogares de bajos ingresos (en promedio,

FIGURA 2. Análisis de sensibilidad: variación del costo por año de vida salvado (AVS) en función de la incidencia de la infección por Haemophilus influenzae tipo b (Hib)

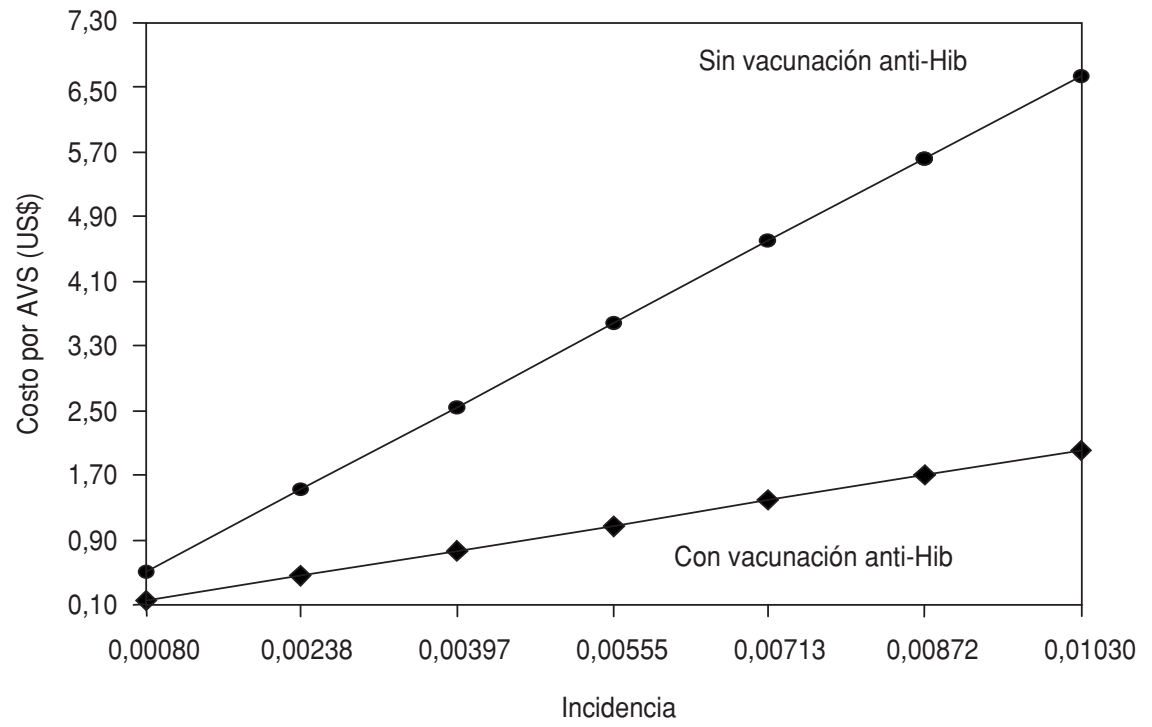

menos de dos salarios mínimos, es decir, US\$200,0 de 2002, aproximadamente). Si estudiáramos poblaciones de mayores ingresos, los costos serían mayores, lo que incrementaría la relación costo-efectividad. Por último, en Colombia se ha cambiado el tipo de vacuna contra Hib utilizado en el programa regular de vacunación: la vacuna monovalente ha sido reemplazada por la vacuna pentavalente (contra la difteria, la tos ferina y el tétano [DPT], la hepatitis B y la infección por Hib), de costo relativamente inferior; esto supone una mayor reducción de los costos directos de vacunación y mayores ahorros para el sistema de salud, lo que incrementa la relación costo-efectividad.

A pesar de estas limitaciones y de que los costos directos e indirectos de la neumonía y la meningitis son en Colombia muy inferiores (hasta 15 veces) a los descritos en países desarrollados (29), se obtiene una relación costo-efectividad de la vacuna muy favorable. Además, los costos por año de vida salvado son proporcionalmente menores que en los países desarrollados. Es evidente, con los supuestos adoptados, que la vacunación sistemática contra Hib ofrece un beneficio social neto importante, a pesar de no haberse valorado y contabilizado todos los beneficios potenciales. Esto concuerda con los resultados de otros estudios donde también se ha demostrado que la vacuna contra Hib (28-31) proporciona beneficios sociales netos. Es importante notar que en esos estudios, el beneficio determinado depende de la incorporación de los costos indirectos. Si solo se contabilizan los costos directos, el resultado se salda con el costo neto de la estrategia de vacunación evaluada.

Una de las lecciones que es preciso aprender es la necesidad de instaurar en Colombia un sistema de información sanitaria integral, que capture la información epidemiológica, así como la relacionada con la aplicación de los recursos destinados a las actividades de los sistemas de salud. Ello impone la tarea de desarrollar, en las instituciones públicas y privadas, sistemas de costos que faciliten la recopilación 
de información para estudios de evaluación de la eficiencia y la equidad de las intervenciones. Además, los países en desarrollo deben adoptar y desarrollar sistemas de cuentas nacionales de salud que reflejen los esfuerzos públicos y privados para el financiamiento del sistema sanitario. Sin crear estas condiciones, en las valoraciones económicas que actualmente se realizan, incluida la nuestra, se corre el riesgo de omitir costos económicos relacionados con la perdida de productividad generada por la carga de enfermedad que plantea el perfil epidemiológico de cada país.

Por otro lado, el fortalecimiento de un sistema de vigilancia adecuado de la meningitis y la neumonía en Colombia permitiría disponer de información oportuna sobre los patógenos, la población sometida a un riesgo particularmente alto de contraer la enfermedad, las tendencias de la resistencia antimicrobiana y otros elementos indispensables para la planificación de medidas para proteger la salud de la población. En Colombia, la deficiencia del sistema de información sanitaria respecto de estas infecciones, notoria desde 1997, genera vacíos de información que hacen más inciertos los supuestos de programación de recursos, al no poder caracterizar adecuadamente los casos ni prever las intervenciones más adecuadas.

El análisis de costo-efectividad como método de evaluación económica es cada vez más útil para visualizar las razones técnicas que permiten racionalizar el uso de los recursos y favorecer los propósitos de eficiencia que se plantean en la mayoría de las instancias de decisión. El análisis de costo-efectividad ha sido la técnica más utilizada para evaluar económicamente la incorporación de vacunas a los programas ordinarios de los países desarrollados. Sin embargo, en los países en desarrollo, tales decisiones responden más a imposiciones de organismos de cooperación o fabricantes de productos biológicos que a estudios propios en los que se haya demostrado la necesidad de introducir la vacuna en cuestión. Esto se debe principal- mente a la falta de personas entrenadas para realizar dichos estudios y de datos adecuados, e impide conocer los verdaderos impactos de la adopción de vacunas en los sistemas de salud.

Por último, es necesario reconocer la escasez de datos de evaluaciones económicas de intervenciones, como la descrita en este artículo o de otro tipo, en algunos países de la Región de las Américas. Por esta razón, se debe promover el uso de estas herramientas, especialmente antes de introducir nuevas tecnologías biomédicas en los sistemas de salud, ya que en la mayoría de los países de la Región, los recursos para cubrir los costos del sector sanitario son cada vez más limitados, lo que exige aplicarlos con criterios de eficiencia y equidad. En tal sentido, los resultados del presente estudio deben tenerse en cuenta para tomar decisiones de políticas públicas dirigidas a lograr a corto plazo coberturas útiles de vacunación contra Hib en Colombia, según su impacto sobre la morbilidad y, la mortalidad y los ahorros potenciales que genera esta estrategia.

\section{REFERENCIAS}

1. Organización Panamericana de la Salud. Enfermedades transmisibles. Control de las infecciones respiratorias agudas. Washington, D.C.: OPS; 1997

2. World Health Organization. World Health Report 2003: shaping the future. Geneva: WHO; 2003.

3. Frank S, Mark CS. Vaccines for children in rich and poor countries. Lancet. 1999;354 (suppl. II):7-11.

4. Shapiro ED. Infections caused by Haemophilus influenzae type $b$. The beginning of the end? JAMA. 1993;269:210-4.

5. Limcango MR, Armour CL, Salole EG, Taylor SJ. Cost-benefit analysis of a Haemophilus influenzae type $\mathrm{b}$ meningitis prevention programme in the Philippines. Pharmacoeconomics. 2001;19(4):391-400.

6. Loguercio M, Grisi S, De Ulloa EA. Aspectos epidemiológicos de la infección por Haemophilus Influenzae Tipo b. Rev Panam Salud Publica. 2000;7(5):332-8.

7. Colombia, Ministerio de Salud. Documento marco. Primera Jornada Nacional de Vacunación para el control y prevención de las enfermedades producidas por Haemophilus influenzae tipo b. Santa Fe de Bogotá; MINSA; 1998.
8. Organización Panamericana de la Salud. Programa Especial para Vacunas e Inmunización (SVI). Reunión para el establecimiento de un sistema de vigilancia epidemiológica en Centro América para Haemophilus influenzae tipo $\mathrm{b}$ y Streptococcus pneumoniae. Managua (Nicaragua): OPS; 1998.

9. Colombia, Ministerio de la Protección Social, Universidad Nacional de Colombia. Situación de salud en Colombia. Informe preliminar. Santa Fe de Bogotá: Universidad Nacional; 2006.

10. Jafari HS, Adams WG, Robinson KA, Plikaytis $\mathrm{BD}$, Wenger JD. Efficacy of Haemophilus influenzae Type $\mathrm{b}$ conjugate vaccines and persistence of disease in disadvantaged populations. Am J Public Health. 1999;89(3):364-8.

11. Monto A, Lehmann D. Acute respiratory infections (ARI) in children: prospects for prevention. Vaccine. 1998;16(16):1582-8.

12. Orenstein WA, Hinman AR. The inmunization system in the United States: the role of school inmunization laws. Vaccine. 1999;17 (Supl 3):19-24.

13. Lederber J, Slope R. Emerging infections. Washington, D.C.: National Academy Press; 1992.

14. Booy R, Hodgson S, Carpenter L, MyonWhite RT, Slack MP, Macfarlane JA, et al. Ef- ficacy of Haemophilus influenzae type b conjugates vaccine PRP-T. Lancet. 1994;344:362-6.

15. Booy R, Heath PT, Slack MPE, Begg N, Moxon ER. Vaccine failures after primary immunization with Haemophilus influenzae type-b conjugate vaccine without booster. Lancet. 1997; 349:1197-202.

16. Lipsitch M. Bacterial vaccines and serotype replacement: lessons from Haemophilus influenzae and prospects for Streptococcus pneumoniae. Emerg Infect Dis. 1999;5:1-16.

17. Khin W, Myo A, Han H, Assad S, Hans B. Comparison of separate and mixed administration of DTPw-HBV and Hib vaccines: immunogenicity and reactogenicity profiles. Int J Infect Dis. 1997;2(2):79-84.

18. Agudelo C, Muñoz N, De la Hoz F. Evaluación rápida del impacto de la vacuna contra Haemophilus influenzae tipo b (Hib) en Colombia. Rev Panam Salud Publica. 2000;8(3):181-93.

19. Higuera A, De La Hoz F, Pastor D, Sarmiento C. Evaluación de la efectividad de la vacuna anti-Haemophilus influenzae en las neumonías bacterianas. Protocolo de investigación. Santa Fe de Bogotá: Instituto Nacional de Salud de Colombia; 1999.

20. De la Hoz F, Higuera AB, Di Fabio JL, Luna M, Naranjo AG, de la Luz Valencia M, et al. 
Effectiveness of Haemophilus influenzae type $\mathrm{b}$ vaccination against bacterial pneumonia in Colombia. Vaccine. 2004;23(1):36-42.

21. Drummond MF, O'Brien BJ, Stoddart GL, Torrance GW. Métodos para la evaluación económica de los programas de asistencia sanitaria. Madrid: Díaz de Santos; 2001.

22. World Health Organization. Estimating the potential cost-effectiveness of using Haemophilus influenzae type $\mathrm{b}(\mathrm{Hib})$ vaccine. Geneva: WHO; 2001. Hallado en: http://www.who. int/vaccines-documents / DocsPDF01 / www654.pdf. Acceso el 4 de junio de 2005.

23. World Health Organization. Introducing Haemophilus influenzae type $\mathrm{b}$ vaccine into immunization programmes. Geneva: WHO; 2001. Hallado en: http://www.who.int/ vaccines-documents/DocsPDF99/www9940. pdf. Acceso el 30 de julio de 2005.

24. World Health Organization. Haemophilus influenzae type $\mathrm{b}$ immunization. Introducing Haemophilus influenzae type $\mathrm{b}(\mathrm{Hib})$ conjugate vaccine into national immunization services. Geneva: WHO; 2001. Hallado en: http:// www.who.int/vaccines-documents/Docs PDF01/www599.pdf. Acceso el 18 de agosto de 2005.
25. Weinstein MC, Siegel JE, Gold MR, Kamlet MS, Russell LB. Recommendations of the panel on cost-effectiveness in health and medicine. JAMA. 1996;276:1253-8.

26. Rodríguez J, Gallardo M. Mortalidad y años de vida ajustados por discapacidad como medidas de la carga de enfermedad. Colombia 1985-1995. Ministerio de Salud, Colombia, 1999.

27. Alvis N, De la Hoz F, Higuera AB, Pastor D, Di Fabio JL. Costos económicos de las neumonías en niños menores de dos años de edad, en Colombia. Rev Panam Salud Publica. 2005;8(3):181-93.

28. Alvis N. Evaluación económica de vacunas: coste efectividad de la vacuna de Hib en niños menores de dos años de edad en Colombia [tesis doctoral]. Valencia (España): Universidad Politécnica de Valencia; 2003.

29. Zhou F, Bisgard KM, Yusuf HR, Deuson RR Bath SK, Murphy TV. Impact of universal Haemophilus influenzae type $\mathrm{b}$ vaccination starting at 2 months of age in the United States: an economic analysis. Pediatrics. 2002; 110(4):653-61.

30. Limcangco MR, Armour CL, Salole EG, Taylor SJ. Cost-benefit analysis of a Haemophilus influenzae type $\mathrm{b}$ meningitis prevention programme in The Philippines. Pharmacoeconomics. 2001;19(4):391-400.

31. Jiménez FI, Guallar-Castillón P, Rubio Terrés C, Guallar E. Cost-benefit analysis of Haemophilus influenzae type $\mathrm{b}$ vaccination in children in Spain. Pharmacoeconomics. 1999;15(1): 75-83.

32. Pokorn M, Kopac S, Neubauer D, Cizman M. Economic evaluation of Haemophilus influenzae type $b$ vaccination in Slovenia. Vaccine. 2001;19(25-26):3600-5

33. Miller MA, McCann L. Policy analysis of the use of hepatitis B, Haemophilus influenzae type $\mathrm{b}-$, Streptococcus pneumoniae conjugate and rotavirus vaccines in national immunization schedules. Health Econ. 2000;9(1):19-35.

Manuscrito recibido el 14 de septiembre de 2005. Aceptado para publicación, tras revisión, el 11 de mayo de 2006.

ABSTRACT Objective. Conjugate vaccines are the best public health tools available for preventing most invasive diseases caused by Haemophilus influenzae type $b$ (Hib), but the high cost of the vaccines has so far kept them from being introduced worldwide. The objective of this study was to estimate the cost-effectiveness of introducing Hib conjugate vaccines for the prevention of meningitis and pneumonia among children under 2 years of age in Colombia.

Methods. We estimated the direct and indirect costs of managing in-hospital pneumonia and meningitis cases. In addition, following the recommendations of the World Health Organization, we assessed the cost-effectiveness of Hib vaccination programs. We also estimated the costs for preventing Hib cases, and the cost per year of life saved in two hypothetical situations: (1) with vaccination against Hib (with $90 \%$ coverage) and (2) without vaccination.

Results. The average in-hospital treatment costs were US\$ 611.50 (95\% confidence interval $(95 \% \mathrm{CI})=$ US\$ 532.2 to US\$ 690.8) per case of pneumonia and US\$ 848.9 (95\% CI = US\$ 716.8 to US\$ 981.0) per case of meningitis. The average cost per Hib case prevented was US\$ 316.7 (95\% CI = US\$ 294.2 to US\$ 339.2). In terms of costeffectiveness, the cost would be US\$ 2.38 per year of life saved for vaccination, versus US\$ 3.81 per year of life saved without vaccination.

Conclusion. Having an adequate Hib vaccination program in Colombia could prevent around 25000 cases of invasive disease per year, representing a cost savings of at least US $\$ 15$ million annually. Furthermore, the program could prevent some 700 deaths per year and save 44054 years of life per year.

Key words Haemophilus influenzae type b, immunization, health resources, cost-benefit analysis, Colombia. 